\title{
Detection of retinal hemorrhages in the presence of blood vessels
}

\author{
Garima Gupta ${ }^{1}$, Keerthi Ram ${ }^{2}$, S.Kulasekaran ${ }^{2}$, Niranjan Joshi ${ }^{2}$, \\ Mohanasankar Sivaprakasam ${ }^{1,2}$ and Rashmin Gandhi ${ }^{3}$ \\ 1 Indian Institute of Technology Madras (IITM), India \\ 2 Healthcare Technology Innovation Centre, IITM, India \\ 3 Centre for Sight, India
}

\begin{abstract}
Segmentation of hemorrhages helps in improving the efficiency of computer assisted image analysis of diseases like diabetic retinopathy and hypertensive retinopathy. Hemorrhages are blood leakages lying in close proximity to blood vessels, which makes their delineation from blood vessels challenging. We use multiresolution morphological processing with a view of achieving perceptual grouping of the hemorrhagic candidates occurring in variable shapes, sizes and textures. We propose a novel method of suppression of candidates lying on blood vessels while attaining a good segmentation of true hemorrhages including the ones attached to the vessels. Evaluated on 191 images having different degrees of pathological severity, our method achieved $>82 \%$ sensitivity at $<7$ false positives per image (FPPI). We further observe that the sensitivity is higher for candidates with bigger sizes.
\end{abstract}

\section{Introduction}

Retinal hemorrhages are signs of retinal disease or injury, observable as dark patches indicative of bleeding. On a retinal image, hemorrhages appear as dark, reddish structures of various sizes: dot hemorrhages are small isolated red spots, blot hemorrhages are irregular, dotted textured, localized circular structures while flame shaped hemorrhages are fan-shaped bleed lesions oriented towards the optic nerve head.

Hemorrhages are a clinical sign of diseases like diabetic retinopathy, and hypertensive retinopathy. Clinical guidelines specify that the location and extent of hemorrhages is a direct indicator of the severity of disease [1]. Detection and segmentation of hemorrhages is therefore an important component for computerassisted screening and grading.

\section{$1.1 \quad$ Prior work}

The most common approach for detection of hemorrhages uses morphological processing for selecting candidates, followed by region growing and supervised classification for rejecting false candidates. In [2] a morphological linear top hat method is used for detecting dot hemorrhage, and is adapted to multiple scales 


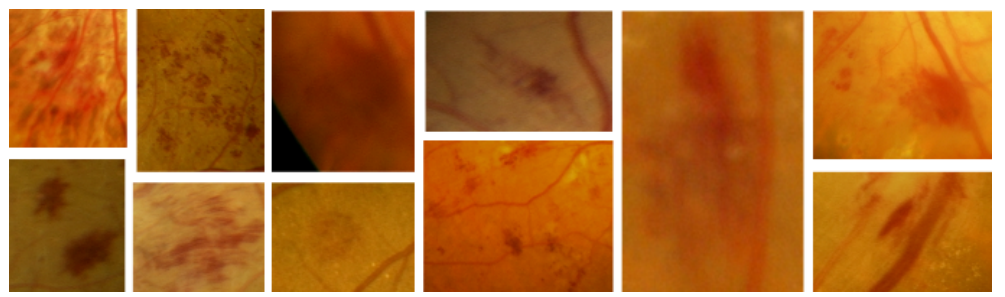

Fig. 1. The irregular shape of blot hemorrhages and streak shape of flame hemorrhages at different sizes presents wide variability in the appearance of large hemorrhages, making their characterization, detection and segmentation a non-trivial task.

for detecting larger hemorrhages. The method uses the complement image and performs multiple linear top-hats for progressively de-enhancing vessels in each scale. Candidates are detected by thresholding and region-growing. The method also performs local vessel detection in the vicinity of candidate and discontinuity assessment. In [3] a multiscale morphological reconstruction method is used for finding local minima, followed by identification of lesion area by dilation with incrementally sized structuring elements and study of edge slope variation. Acharya et al. [4] performed morphological opening with optimally adjusted structuring elements for extracting vasculature separately, and dark structures including hemorrhage and vessels. Hemorrhage candidates were obtained by subtraction followed by denoising.

Among the methods which do not use morphological approach for candidate detection are a multi-scale Gaussian matched-filter with entropy thresholding used in [5] for detection of hemorrhages. In [6] candidate detection is done by normalized cross correlation template matching with a circular template at different radii, followed by region growing to extract hemorrhage candidates. A recent method for large hemorrhage detection [7] uses gradient scale-space and watershed transform, performs partitioning of image regions, and identifies partitions corresponding to hemorrhages using supervised learning.

Hemorrhages can be described using two main characteristics: dark appearance (low intensity) and irregular, amorphous shape. However, intensity in hemorrhages is similar to vessel structures (and fovea), and polymorphic shape of hemorrhages makes exhaustive modeling intractable. It has been reported in literature that vessels and fovea are the two main false alarms in hemorrhage detection [6]. Hemorrhages being a sign of blood-related pathology, might present themselves in close proximity to vessels, and sometimes overlap or occlude them. Though much research has been directed at blood vessel detection, the performance of published algorithms on pathological cases has not been established, especially when there are hemorrhages which appear connected to vessels [8].

In this work we address the detection and segmentation of hemorrhages showing wide variability in size, shape and appearance, including irregular shaped hemorrhages, blots, flames, bleeding and hemorrhages attached to vessels. We use multi-scale processing method for simplifying the characterization of hemorrhage types. Our approach proposes a novel method for rejection of false alarms 
on blood vessels using a filter-set based on morphometric properties, and segmentation of hemorrhages by removing curvilinear parts of detected candidates which could actually be vessel. We also use a set of novel features derived from vesselness analysis within each candidate.

\section{Our approach}

Our approach builds a multiscale morphological pyramid followed by carrying out operations which take place in each scale (candidate detection, false alarm rejection), merging of candidates from different scales, refinement of segmentation(resolving adjoined hemorrhages, pruning vessels in regions), and region ranking as shown in fig. 2 .

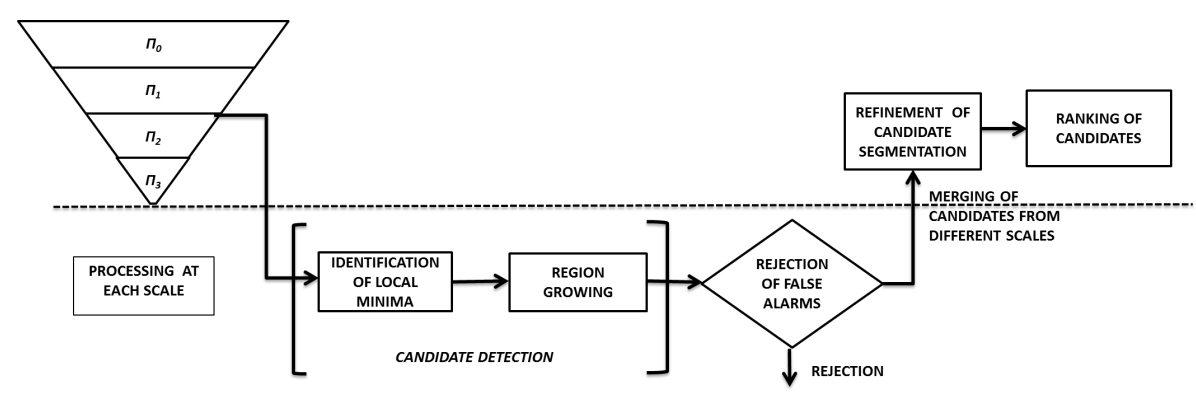

Fig. 2. Flow diagram of our proposed approach

\subsection{Candidate detection}

We construct a multiscale morphological pyramid (fig.3) $\Pi$, where the level0 of the pyramid is the opening of green channel $I_{g}$, and each following level stores a successive opening (with resampling). We use multi-scale morphological opening pyramid for homogenizing the freckled texture within the hemorrhage and improving the edge definition of the object of interest in scale space.

Region-growing segmentation: At each scale, we identify the centroids of the connected set of pixels with same intensity, surrounded by pixels having greater intensity as local minima. The local minima with the intensity lying in the darkest $10 \%$ range of the contrast-normalized image are used as initialization points for region growing process.

We use an iterative intensity-based region growing where at each iteration the region adds neighbor pixels whose intensity is within a certain contrast limit from the current region mean. At each scale of $\Pi$, the local contrast is estimated and normalized by dividing local background $\left(B g_{i}\right)$ from $\Pi_{i}$. The contrast-normalized image, $P c_{i}=\frac{\Pi_{i}}{B g_{i}}-1$ enables the use of a constant parameter for contrast while region-growing. 


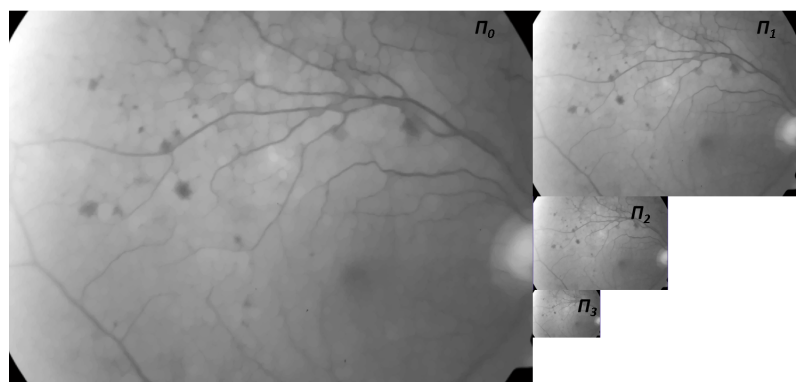

Fig. 3. Morphological opening pyramid shown for 4 levels. Contrast of edges becomes stronger and fuzzy regions within the lesion become better homogenized in scale space

For restricting the growth of vessel seeds into branches and bends, we set stopping criteria based on the displacement of the center of mass of the currently growing region. Due to downsampling, the resolution of the branching angles of the vessels decreases. The opening operation with a disk structuring element merges the close-by lying vessels especially the branches of the vessels. Thus we set stopping criteria based on instantaneous region structure in every growth step, and examine morphological attributes of the region grown so far: solidity, major axis length and minor axis length to restrict the region from growing into the merged bifurcations.

\subsection{Rejection of false alarms}

For performing rejection of vascular false alarms in $E_{i}$, we examine each grown candidate by a sequence of morphological tests based on five key properties: eccentricity $\varepsilon$, width $\eta$, elongation $\kappa$, aspect ratio $\gamma$ and truncation. Elongation $\kappa$ is defined as the standard deviation of Euclidean distances of the perimeter pixels from centroid of the candidate. Truncation (VTR) is quantified by the number of vessel pixels removed from the candidates by morphological opening with a line structuring element oriented in the direction of minor axis and its length dependent on minor axis length of the object. Apart from false alarm rejection, VTR helps in pruning the candidates by removing the vessel pixels.

Table 1. Table showing the percentage of lesions and vessels retained in each false alarm rejection stage (percentage of each stage is calculated separately)

\begin{tabular}{ccccccc}
\hline & & VRS-1 & ELA & VRS-2 & VTR & \\
\hline & Condition & High $\boldsymbol{\varepsilon}$, & $\boldsymbol{\kappa} \times \boldsymbol{\gamma}<$ & Very & & Total \\
& & small $\boldsymbol{\eta}$ & $\mathbf{1 0}$ & small $\boldsymbol{\eta}$ & & \\
\hline True hemorrhage & \multirow{2}{*}{ \%retained } & $100 \%$ & $97 \%$ & $63 \%$ & $100 \%$ & $99 \%$ \\
Blood vessel & $63 \%$ & $61 \%$ & $78 \%$ & $96 \%$ & $56 \%$ \\
\hline Vessels per lesion & 9.42 & & & & & 5.375 \\
\hline
\end{tabular}

Table 1 shows the conditions used in the sequential stages of false alarm rejection: VRS-1 (vessel rejection 1), ELA (elongation analysis), VRS-2 (ves- 
sel rejection 2), and VTR. Candidates passing ELA are not scrutinized further, whereas those failing the condition are checked against VRS-2 and VTR successively. The table indicates the percentage of true hemorrhages and blood vessels retained by each stage of rejection, and the number of vessels retained per true hemorrhage. In a sample subset of 16 images having manual vessel annotation, the number of vessels per hemorrhage in $E_{i}$ was 9.42 . At the end of the sequential rejection, the vessels per hemorrhage drops to 5.375 ( $44 \%$ vessel rejection), for a $99 \%$ retention of true hemorrhages.

\subsection{Merging and Refinement of candidate segmentation}

The above steps for candidate detection and false-alarm rejection operate on each level of the pyramid. The regions from all the levels are merged using logical OR operation after resampling the binary outputs to a common size (size of $\Pi_{1}$ ). This step while ensuring accumulation of all possible candidates, could potentially lead to altering the shape of candidates, adjoint candidates merging into one, and vessel regions clumping with candidates. Such candidates are selected by measuring their area and solidity(area to convex area ratio). Within these candidates, the locations where the cross sectional widths change, are identified by morphological operation. We perform top-hat morphological operation with linear structuring element rotated in different orientations at a resolution of $10^{\circ}$. The pixels which get removed in more than five orientations belong to the non-curvilinear part of the candidates.

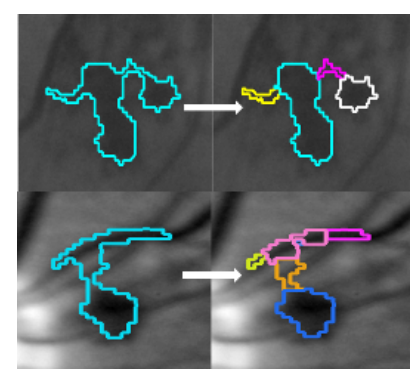

Fig. 4. Top-left and bottom-left subimages show hemorrhage candidates with their corresponding refinement of candidate segmentation stage output.

\subsection{Candidate ranking}

We use a supervised learning technique for assigning confidence scores to each candidate in the range of 0 to 1 . At each candidate, we extract a set of features and the ranking method learns a mapping between the feature values to the confidence score. We train a two-class statistical classifier such as random forest [9], and use the posterior probability of the 'Hemorrhage' class as predicted confidence score. Table 2 lists the different types of features we extract at each 
candidate. Among the novel features we use, mean vesselness in the candidate (found by Frangi filter, Gabor filtering, anisotropic diffusion filtering), tortuosity of the candidate after skeletonization, standard deviation of ridgeline orientation within the candidate, and the weighted sum of histogram of ridgeline orientations weighted about $0^{\circ}$ with signum function over angles are specifically targeted at discriminating vascular candidates.

Table 2. LIST OF FEATURES

\begin{tabular}{ll}
\hline Feature & Description \\
\hline Color & Difference between the mean pixel values inside the ob- \\
& ject and in a circular region around the candidate, RGB. \\
& Color within and on boundary in opponency images. \\
\hline Filter Banks & Gaussian filter Bank: $G(\sigma), G_{x}(\sigma), G_{y}(\sigma), G_{x x}(\sigma), G_{y y}(\sigma)$ \\
& and $G_{x y}(\sigma)$, green channel. \\
& DOG filter Bank: $\sigma_{1}=4,8,12$ and $\sigma_{0}=\left(\sigma_{1} / 3\right)+1, \mathrm{RGB}$. \\
\hline Texture & Statistics of Gray-Level co-occurrence matrix. \\
& Local range,standard deviation,entropy of each candidate \\
& pixel within a window in green channel. \\
& Schmid Filter Bank: 13 rotationally invariant filters. \\
\hline Edge Strength & Gradient magnitude response \\
\hline Vessel Enhancement & Vessel Enhancement by Frangi vesselness, Gabor filter, \\
\hline Tortuosity Measure & Anisotropic Non-linear Diffusion filtering. \\
\hline Morphological Properties & Found using candidate skeleton. \\
\hline Orientation-based & Minor axis length. \\
\hline & Standard deviation of orientation inside candidate. \\
& Ratio of eigenvalues obtained from Hessian matrix. \\
& Weighted sum of histogram of orientations.
\end{tabular}

In addition to the features shown in Table 2, we compute a set of features based on transverse profile modelling of the candidate centreline. We find the centreline traversing through the candidate connecting farthest points on its perimeter, by an explorative shortest path algorithm.

We use a Gaussian function to model the transverse profiles of the candidate at discrete points on the centreline. The parameter $\sigma$ of the Gaussian approximates the transverse width at the centreline. From the transverse fits, we extract the distribution of $\sigma$, and find statistical measures like mode, mean, standard deviation, kurtosis, second moment about mode. These features are specifically aimed at discriminating candidates with vascular structure from thick flames and hemorrhages.

Training: We trained a random forest classifier with true and false hemorrhage candidates selected from two local datasets having resolutions $2240 \times 1488$ and $1280 \times 1024$. Totally 191 images were obtained from 58 diabetic subjects in a clinical setting using dilatation, and 2008 hemorrhage regions were annotated by a clinician. 


\section{Results and discussion}

Using the set of 191 images, we performed 10-fold cross validation to sweep an FROC curve, varying thresholds on the confidence score. Positives are candidates having confidence score surpassing the threshold. True-positives are positives overlapping with the expert-marked ground truth annotation while falsepositives do not overlap with the ground truth.
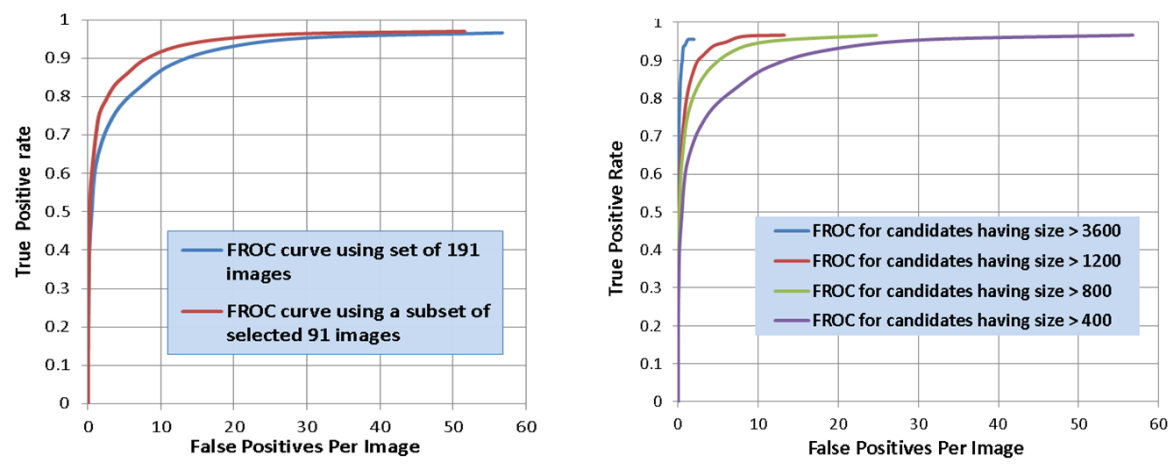

Fig. 5. (a)FROC curve - Most hemorrhage literature usually report ROC curve conveying performance of image-level decision(presence of hemorrhage). FROC is more apt to express hemorrhage detection performance. (b)FROC for varying candidate sizes.

Our method achieves $82 \%$ sensitivity at 6.9 FPPI for 191 images, which includes different degrees of pathology, and complications in the form of choroidal vessels, proliferative vessels and laser marks. Considering a subset of 91 images, selected based on absence of the above complications, we observed performance of $86 \%$ sensitivity at 5.5 FPPI. This can be contrasted with the performance of $85 \%$ sensitivity, 4 FPPI reported in [6]. Similar to the area analysis presented in [6], we studied the performance among various sizes of candidates. From the resulting FROC curves (fig.5) we see that among the higher sized candidates, the detection sensitivity is higher.

The wide variability in the appearance, color, texture, and morphological properties among hemorrhages has resulted in near-uniform distribution of confidence scores on true hemorrhage candidates. The vessel rejection nature of the features can be seen from the confidence value distribution of candidates lying on the blood vessels (fig.6) which demonstrates that $>90 \%$ of them have confidence value $\leq 0.2$.

\section{Conclusion}

Our approach presents a high-quality hemorrhage detection algorithm where unlike previous published works, we use pre-emptive region growing technique 

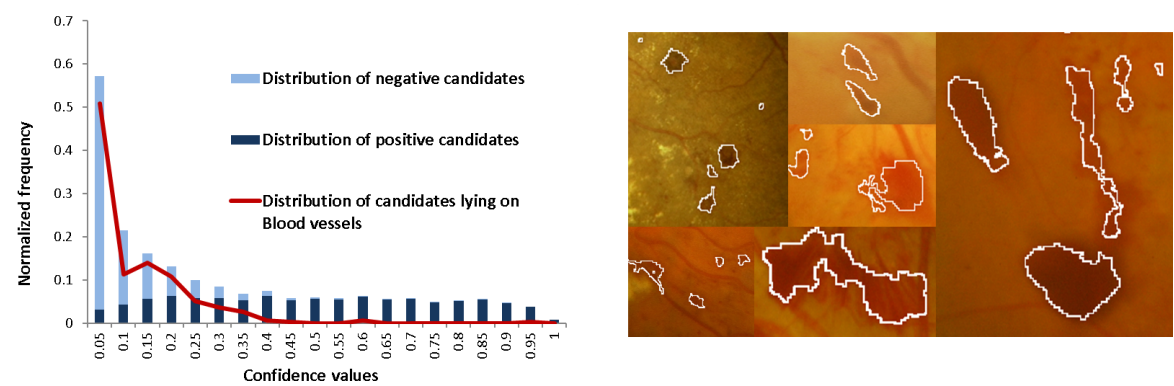

Fig. 6. (a) Distribution of confidence scores among the positive and negative candidates. The overlaid curve in red illustrates the confidence value distribution of the candidates lying on the blood vessel ground truth (marked by an expert ophthalmologist for 16 images).(b)Subimages showing detected positive hemorrhagic regions

to prevent the candidates from growing too much into vessels, structure-based properties for rejection of blood vessel candidates while retaining hemorrhagic candidate, and a set of novel features based on vesselness within the candidates to successfully discriminate vessels from hemorrhages. The performance achieved is comparable with the state of art. Based on our experiments, we can infer that our method provides higher sensitivity of detection. Visual inspection indicates good segmentation of big hemorrhages. We plan to use higher level image information for the suppression of macula(the other prominent confounder), in our future work.

\section{References}

1. Fleming, A.D., Goatman, K.A., Philip, et al.: The role of haemorrhage and exudate detection in automated grading of diabetic retinopathy. BJO 94(6) (2010) 706-711

2. Fleming, A., Goatman, K., Williams, G., Philip, et al.: Automated detection of blot haemorrhages as a sign of referable diabetic retinopathy. (2008) 4-8

3. Zhang, X., Fan, G.: Retinal spot lesion detection using adaptive multiscale morphological processing. (2006) 490-501

4. Acharya, U., Lim, C., Ng, et al.: Computer-based detection of diabetes retinopathy stages using digital fundus images. Proc. of the IME 223(5) (2009) 545-553

5. Kande, G.G.B., Savithri, T.S., Subbaiah, P.V.: Automatic Detection of Microaneurysms and Hemorrhages in Digital Fundus Images. Journal of Digital Imaging 23(4) (2009) 430-7

6. Bae, J.P., Kim, K.G., Kang, et al.: A study on hemorrhage detection using hybrid method in fundus images. Journal of digital imaging 24(3) (2011) 394-404

7. Tang, L., Niemeijer, M., Reinhardt, et al.: Splat feature classification with application to retinal hemorrhage detection in fundus images. IEEE Trans. Med. Img. 32(2) (2013) 364-375

8. Garcia, M., Sanchez, C., Lopez, M.I., et al.: Automatic detection of red lesions in retinal images using a multilayer perceptron neural network. (2008) 5425-5428

9. Breiman, L.: Random forests. Machine Learning 45(1) (2001) 5-32 